\title{
AN ANALYTICAL INVESTIGATION OF A 2D-PPMS HOLLOW INFINITE CYLINDER UNDER THERMO-ELECTRO-MECHANICAL (TEM) LOADINGS
}

\author{
Mohsen Meshrini \\ School of Science and Engineering, Sharif University of Technology, International Campus, Kish Island, Iran \\ e-mail:meshkini@kish.sharif.edu
}

KeIKHOSROW FiroozBAKHSH

Department of Mechanical Engineering, Sharif University of Technology, Tehran, Iran

e-mail: firoozbakhsh@sharif.edu

MOHSEN JABBARI

Department of Mechanical Engineering, South Tehran Branch, Islamic Azad University, Iran

e-mail:m_jabbari@azad.ac.ir

Ali SelkGhafari

School of Science and Engineering, Sharif University of Technology, International Campus, Kish Island, Iran

e-mail: a_selkgafari@sharif.edu

\begin{abstract}
The analytical solution of steady-state asymmetric thermo-electro-mechanical loads of a hollow thick infinite cylinder made of porous piezoelectric materials (2D-PPMs) based on two-dimensional equations of thermoelasticity is considered. The general form of thermal and mechanical boundary conditions is considered on the inside and outside surfaces. A direct method is used to solve the heat conduction equation and the non-homogenous system of partial differential Navier equations using the complex Fourier series and the powerexponential law functions method. The material properties are assumed to depend on the radial and circumferential variable and are expressed as power-exponential law functions along the radial and circumferential direction.
\end{abstract}

Keywords: piezoelectric, porothermoelastisity, 2D-PPMs, hollow cylinder, TEM

\section{Introduction}

Porous piezoelectric materials (PPMs) have lower acoustic impedance and can be incorporated in medical ultrasonic imaging devices. They are widely used for applications such as low frequency hydrophones, accelerometers, vibratory sensors and contact microphones. The classical method of analysis is to combine equilibrium equations with stress-strain and strain-displacement relations to arrive at governing equations in terms of displacement components, namely the Navier equations (Hetnarski and Eslami, 2009). Li et al. (2003) presented fabrication and evaluation of porous piezoelectric ceramics and poroussity-graded piezoelectric actuators. Zielinski (2010) discussed the fundamentals of multi physics modeling of piezo-poro-elastic structures. The processing and properties of porous piezoelectric materials with high hydrostatic figures of merit was given by Bowen et al. (2004). The porous piezoelectric composites with extremely high reception was discussed by Topolov and Turik (2001). Ciarletta and Scarpetta (1996) gave some results on thermoelasticity for porous piezoelectric materials. Batifol et al. (2007) presented a finite-element study of a piezoelectric/poroelastic sound package concept. Zeng et al. (2007) have discussed the processing and piezoelectric properties of porous PZT ceramics. Ivanov et al. (2002) used the porous piezoelectric ceramics materials for ultrasonic flaw detection and medical 
diagnostics. Ding et al. (2004) presented an analytical solution of a special non-homogeneous pyroelectric hollow cylinder for piezothermoelastic axisymmetric plane strain dynamic problems. Akbari Alashti et al. (2013) presented thermo-elastic analysis of a functionally graded spherical shell with piezoelectric layers by differential quadrature method. Jabbari et al. $(2012,2016)$ studied mechanical and thermal stresses in FGPPM hollow cylinders. Meshkini et al. (2017) studied a asymmetric mechanical and thermal stresses in 2D-FGPPMs hollow cylinder. The applied separation of variables and the complex Fourier series to solve the heat conduction and Navier equations.

In this study, an analytical method is presented for mechanical and thermal stress analysis for a hollow infinite cylinder made of fluid saturated porous piezoelectric materials (2D-PPMs). In present study, the material properties are assumed to be expressed by power functions in the radial and circumferential direction. The effects of compressibility, pore volume fraction (porosity), and electric potential coefficient on displacements, electric potential and stresses are studied. Temperature distribution is considered in the steady state asymmetric case and mechanical and thermal boundary conditions by satisfying the stress and displacement boundary condition.

\section{Governing equations}

\subsection{Stress analysis}

The strain-displacement relations and electric intensity are (Ding et al., 2004)

$$
\begin{aligned}
& \varepsilon_{r r}=\frac{\partial u}{\partial r} \quad \varepsilon_{\theta \theta}=\frac{1}{r} \frac{\partial v}{\partial \theta}+\frac{u}{r} \quad \varepsilon_{r \theta}=\frac{1}{2}\left(\frac{1}{r} \frac{\partial u}{\partial \theta}+\frac{\partial v}{\partial r}-\frac{v}{r}\right) \\
& E_{r}=\frac{\partial \psi}{\partial r} \quad E_{\theta}=\frac{1}{r} \frac{\partial \psi}{\partial \theta}
\end{aligned}
$$

Stress-strain relations of a 2D-PPM cylinder for the asymmtric condition are (Meshkini et al., 2017)

$$
\begin{aligned}
& \sigma_{r r}=C_{11} \varepsilon_{r r}+C_{12} \varepsilon_{\theta \theta}+e_{21} E_{r}-\gamma p-C_{1}^{T} T(r, \theta) \\
& \sigma_{\theta \theta}=C_{12} \varepsilon_{r r}+C_{22} \varepsilon_{\theta \theta}+e_{22} E_{r}-\gamma p-C_{2}^{T} T(r, \theta) \\
& \sigma_{z z}=C_{12}\left(\varepsilon_{r r}+\varepsilon_{\theta \theta}\right)+e_{23} E_{r}-\gamma p-C_{3}^{T} T(r, \theta) \\
& \sigma_{r \theta}=2 C_{44} \varepsilon_{r \theta}+e_{24} E_{\theta} \quad D_{r r}=e_{21} \varepsilon_{r r}+e_{22} \varepsilon_{\theta \theta}-\varepsilon_{22} E_{r}+g_{21} T(r, \theta) \\
& D_{\theta \theta}=2 e_{24} \varepsilon_{r \theta}-\varepsilon_{21} E_{\theta}+g_{22} T(r, \theta)
\end{aligned}
$$

where $p$ is related to Biot's modulus, volumetric strain and the variation of the fluid content. Considering the undrained conditions $(\xi=0)$ as (Jabbari et al., 2012)

$$
p=M\left(\xi-\gamma\left(\varepsilon_{r r}+\varepsilon_{\theta \theta}\right)=-M \gamma\left(\varepsilon_{r r}+\varepsilon_{\theta \theta}\right)\right.
$$

Using relations (2.2) and (2.3), the stress-strain relations of the 2D-PPM for the asymmtric condition are (Meshkini et al., 2017)

$$
\begin{aligned}
\sigma_{r r} & =\widehat{C}_{11} \varepsilon_{r r}+\widehat{C}_{12} \varepsilon_{\theta \theta}+e_{21} E_{r}-C_{1}^{T} T(r, \theta) \\
\sigma_{\theta \theta} & =\widehat{C}_{12} \varepsilon_{r r}+\widehat{C}_{22} \varepsilon_{\theta \theta}+e_{22} E_{r}-C_{2}^{T} T(r, \theta) \\
\sigma_{z z} & =\widehat{C}_{12}\left(\varepsilon_{r r}+\varepsilon_{\theta \theta}\right)+e_{23} E_{r}-C_{3}^{T} T(r, \theta) \\
\sigma_{r \theta} & =2 \widehat{C}_{44} \varepsilon_{r \theta}+e_{24} E_{\theta} \quad D_{r r}=e_{21} \varepsilon_{r r}+e_{22} \varepsilon_{\theta \theta}-\varepsilon_{22} E_{r}+g_{21} T(r, \theta) \\
D_{\theta \theta} & =2 e_{24} \varepsilon_{r \theta}-\varepsilon_{21} E_{\theta}+g_{22} T(r, \theta)
\end{aligned}
$$


and

$$
\widehat{C}_{11}=C_{11}+C_{M} \quad \widehat{C}_{12}=C_{12}+C_{M} \quad \widehat{C}_{22}=C_{22}+C_{M} \quad \widehat{C}_{44}=C_{44}
$$

where $C_{M}=M \gamma^{2}$ and $C_{i}^{T}$ are thermal moduli which can be expressed by elastic constants and linear thermal expansion coefficients $\alpha_{i}$ (Ding et al., 2004)

$$
C_{1}^{T}=C_{11} \alpha_{r}+2 C_{12} \alpha_{\theta} \quad C_{2}^{T}=2 C_{12} \alpha_{r}+C_{22} \alpha_{\theta}
$$

under consideration $\alpha_{r}=\alpha_{\theta}=\alpha$ (Hetnarski and Eslami, 2009). Therefore,

$$
C_{1}^{T}=\left(C_{11}+2 C_{12}\right) \alpha \quad C_{2}^{T}=\left(2 C_{12}+C_{22}\right) \alpha \quad C_{3}^{T}=C_{1}^{T}
$$

The equilibrium equations in the radial and circumferential direction, disregarding the body force and the inertia terms, are (Ding et al., 2004)

$$
\begin{array}{ll}
\frac{\partial \sigma_{r r}}{\partial r}+\frac{1}{r} \frac{\partial \sigma_{r \theta}}{\partial \theta}+\frac{1}{r}\left(\sigma_{r r}-\sigma_{\theta \theta}\right)=0 & \frac{\partial \sigma_{r \theta}}{\partial r}+\frac{1}{r} \frac{\partial \sigma_{\theta \theta}}{\partial \theta}+\frac{2}{r} \sigma_{r \theta}=0 \\
\frac{\partial D_{r r}}{\partial r}+\frac{1}{r} \frac{\partial D_{\theta \theta}}{\partial \theta}+\frac{1}{r} D_{r r}=0 &
\end{array}
$$

To obtain the equilibrium equations in terms of displacement components for the 2D-PPM cylinder, the functional relationship of the material properties must be known. Because the cylinder material is assumed to be graded along the radial and circumferential direction, the coefficient of thermal expansion and electric constants are assumed to be described with the power-exponential laws as

$$
\begin{array}{lrl}
\alpha=\alpha_{0} \widetilde{r}^{m_{1}} \mathrm{e}^{n_{1} \theta} & C_{i j}=\bar{C}_{i j} \widetilde{r}^{m_{2}} \mathrm{e}^{n_{2} \theta} & K=k_{0} \widetilde{r}^{m_{3}} \mathrm{e}^{n_{3} \theta} \\
e_{2 i}=\bar{e}_{2 i} \widetilde{r}^{m_{4}} \mathrm{e}^{n_{4} \theta} & \varepsilon_{2 i}=\bar{\varepsilon}_{2 i} \widetilde{r}^{m_{5}} \mathrm{e}^{n_{5} \theta} & g_{2 i}=\bar{g}_{2 i} \widetilde{r}^{m_{6}} \mathrm{e}^{n_{6} \theta}
\end{array}
$$

where $\widetilde{r}=r / a$ and $a$ is the inner radius.

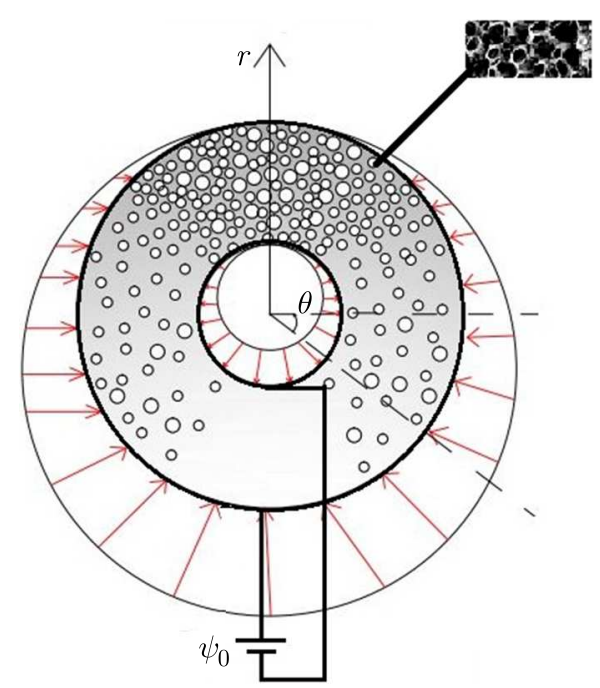

Fig. 1. Geometric model of a 2D-PPM hollow cylinder under two dimensional inner and outer Thermo-Electro-Mechanical (TEM) loads

Using relations (2.4) and (2.9) into (2.8), the Navier equations in terms of the displacement components are 


$$
\begin{aligned}
& u_{, r r}+\left(m_{2}+1+\left(m_{2}-1\right) \frac{\widehat{\bar{C}}_{12}}{\widehat{\bar{C}}_{11}}\right) \frac{1}{r} u, r+\frac{m_{2} \widehat{\bar{C}}_{12}-\widehat{\bar{C}}_{22}}{\widehat{\bar{C}}_{11}} \frac{1}{r^{2}} u+\frac{n_{2} \bar{C}_{44}}{\widehat{\bar{C}}_{11}} \frac{1}{r} v_{, r}-\frac{n_{2} \bar{C}_{44}}{\widehat{\bar{C}}_{11}} \frac{1}{r^{2}} v \\
& +\frac{\bar{C}_{44}}{\widehat{\bar{C}}_{11}} \frac{1}{r^{2}} u_{, \theta \theta}+\frac{n_{2} \bar{C}_{44}}{\widehat{\bar{C}}_{11}} \frac{1}{r^{2}} u_{, \theta}+\frac{\bar{C}_{12}+\bar{C}_{44}}{\widehat{\bar{C}}_{11}} \frac{1}{r} v_{, r \theta}+\frac{m_{2} \bar{C}_{12}-\bar{C}_{22}-\bar{C}_{44}}{\widehat{\bar{C}}_{11}} \frac{1}{r^{2}} v_{, \theta} \\
& +\left(\frac{\bar{e}_{21}}{\widehat{\widehat{C}}_{11}} \psi_{, r r}+\frac{\left(m_{4}+1\right) \bar{e}_{21}-\bar{e}_{22}}{\widehat{\bar{C}}_{11}} \frac{1}{r} \psi_{, r}+\frac{\bar{e}_{24}}{\widehat{\widehat{C}}_{11}} \frac{1}{r^{2}} \psi_{, \theta \theta}+\frac{n_{4} \bar{e}_{24}}{\widehat{\bar{C}}_{11}} \frac{1}{r^{2}} \psi_{, \theta}\right) \widetilde{r}^{m_{4}-m_{2}} \mathrm{e}^{\left(n_{4}-n_{2}\right) \theta} \\
& =\left(\frac{\left(m_{1}+m_{2}+1\right) \bar{C}_{11}+2\left(m_{1}+m_{2}\right) \bar{C}_{12}-\bar{C}_{22}}{\widehat{\bar{C}}_{11}} \frac{1}{r} T+\frac{\bar{C}_{11}+2 \bar{C}_{12}}{\widehat{\bar{C}}_{11}} T_{, r}\right) \alpha_{0} \widetilde{r}^{m_{1}} \mathrm{e}^{n_{1} \theta} \\
& v_{, r r}+\left(m_{2}+1\right) \frac{1}{r} v_{, r}-\left(m_{2}+1\right) \frac{1}{r^{2}} v+n_{2} \frac{\widehat{C}_{22}}{\bar{C}_{44}} \frac{1}{r^{2}} v_{, \theta}+\frac{\widehat{C}_{22}}{\bar{C}_{44}} \frac{1}{r^{2}} v_{, \theta \theta} \\
& +\left(m_{2}+1+\frac{\widehat{C}_{22}}{\bar{C}_{44}}\right) \frac{1}{r^{2}} u_{, \theta}+n_{2} \widehat{\bar{C}}_{12} \frac{1}{\bar{C}_{44}} \frac{1}{r} u_{, r}+\left(1+\frac{\widehat{C}_{12}}{\bar{C}_{44}}\right) \frac{1}{r} u_{, r \theta}+n_{2} \frac{\widehat{\bar{C}}_{22}}{\bar{C}_{44}} \frac{1}{r^{2}} u \\
& +\left(n_{4} \bar{e}_{22} \frac{1}{\bar{C}_{44}} \frac{1}{r} \psi_{, r}+\frac{\bar{e}_{22}+\bar{e}_{24}}{\bar{C}_{44}} \frac{1}{r} \psi_{, r \theta}+\left(m_{4}+2\right) \frac{\bar{e}_{24}}{\bar{C}_{44}} \frac{1}{r^{2}} \psi_{, \theta}\right) \widetilde{r}^{m_{4}-m_{2}} \mathrm{e}^{\left(n_{4}-n_{2}\right) \theta} \\
& =\left(\left(n_{1}+n_{2}\right) \frac{2 \bar{C}_{12}+\bar{C}_{22}}{\bar{C}_{44}} \frac{1}{r} T+\frac{2 \bar{C}_{12}+\bar{C}_{22}}{\bar{C}_{44}} \frac{1}{r} T, \theta\right) \alpha_{0} \widetilde{r}^{m_{1}} \mathrm{e}^{n_{1} \theta} \\
& \psi_{, r r}+\left(m_{5}+1\right) \frac{1}{r} \psi_{, r}+n_{5} \frac{\bar{\varepsilon}_{21}}{\bar{\varepsilon}_{22}} \frac{1}{r^{2}} \psi_{, \theta}+\frac{\bar{\varepsilon}_{21}}{\bar{\varepsilon}_{22}} \frac{1}{r^{2}} \psi_{, \theta \theta}-\left(\frac{\bar{e}_{21}}{\bar{\varepsilon}_{22}} u_{, r r}+\frac{\left(m_{4}+1\right) \bar{e}_{21}+\bar{e}_{22}}{\bar{\varepsilon}_{22}} \frac{1}{r} u_{, r}\right. \\
& \left.+\frac{m_{4} \bar{e}_{22}}{\bar{\varepsilon}_{22}} \frac{1}{r^{2}} u+\frac{n_{4} \bar{e}_{24}}{\bar{\varepsilon}_{22}} \frac{1}{r} v_{, r}-\frac{n_{4} \bar{e}_{24}}{\bar{\varepsilon}_{22}} \frac{1}{r^{2}} v+\frac{\left(m_{4}+1\right) \bar{e}_{22}-\bar{e}_{24}}{\bar{\varepsilon}_{22}}\right) \frac{1}{r^{2}} v_{, \theta} \\
& \left.+\frac{\bar{e}_{24}}{\bar{\varepsilon}_{22}} \frac{1}{r} v_{, r \theta}\right) \widetilde{r}^{m_{4}-m_{5}} \mathrm{e}^{\left(n_{4}-n_{5}\right) \theta}=\left(\frac{\left(m_{6}+1\right) \bar{g}_{21}+n_{6} \bar{g}_{22}}{\bar{\varepsilon}_{22}} \frac{1}{r} T+\frac{\bar{g}_{22}}{\bar{\varepsilon}_{22}} \frac{\partial T}{\partial r}\right. \\
& \left.+\frac{\bar{g}_{22}}{\bar{\varepsilon}_{22}} \frac{1}{r} \frac{\partial T}{\partial \theta}\right) \widetilde{r}^{m_{6}-m_{5}} \mathrm{e}^{\left(n_{6}-n_{5}\right) \theta}
\end{aligned}
$$

Navier equations (2.10) are a non-homogeneous system of partial differential equations with non-constant coefficients.

\subsection{Heat conduction problem}

The first law of thermodynamics for energy equation in the steady-state condition for the 2D-PPM two dimensional cylinder is

$$
\frac{1}{r}\left(k r T_{, r}\right)_{, r}+\frac{1}{r^{2}}\left(k T_{, \theta}\right)_{, \theta}=0 \quad a \leqslant r \leqslant b \quad-\pi \leqslant \theta \leqslant+\pi
$$

where $T(r, \theta)$ is temperature distribution, $k(r, \theta)$ is the thermal conduction coefficient and a comma denotes partial differentiation with respect to the space variable.

The thermal boundary conditions are assumed as

$$
S_{11} T(a, \theta)+S_{12} T_{, r}(a, \theta)=f_{1}(\theta) \quad S_{21} T(b, \theta)+S_{22} T_{, r}(b, \theta)=f_{2}(\theta)
$$

we assume that the non-homogeneous thermal conduction coefficient $k(r, \theta)$ is a power function of the radial and circumferential coordinates $(r, \theta)$ as $k(r, \theta)=k_{0} \widetilde{r}^{m_{3}} \mathrm{e}^{n_{3} \theta}$.

Using the definition for the material properties, the temperature equation becomes

$$
T_{, r r}+\left(m_{3}+1\right) \frac{1}{r} T_{, r}+\frac{1}{r^{2}}\left(n_{3} T_{, \theta}+T_{, \theta \theta}\right)=0
$$


The solution to Eq. (2.13) is written in the form of complex Fourier series, as

$$
T(r, \theta)=\sum_{q=-\infty}^{\infty} T_{q}(r) \mathrm{e}^{\mathrm{i} q \theta}
$$

Substituting Eq. (2.14) into Eq. (2.13), the following equation is obtained

$$
T_{q}^{\prime \prime}(r)+\left(m_{3}+1\right) \frac{1}{r} T_{q}^{\prime}(r)+\frac{1}{r^{2}}\left(\mathrm{i} q n_{3}-q^{2}\right) T_{q}(r)=0
$$

Equation (2.15) is the Euler equation and has solutions in the form of

$$
T_{q}(r)=A_{q} r^{\beta}
$$

Substituting Eq. (2.16) into Eq. (2.15), the following characteristic equation is obtained

$$
\beta^{2}+m_{3} \beta+\left(\mathrm{i} q n_{3}-q^{2}\right)=0
$$

the roots of Eq. (2.17) are

$$
\beta_{q_{1,2}}=\frac{-m_{3}}{2} \mp \sqrt{\frac{m_{3}^{2}}{4}+q^{2}-\mathrm{i} q n_{3}}
$$

Thus

$$
T_{q}(r)=A_{q_{1}} r^{\beta_{q_{1}}}+A_{q_{2}} r^{\beta_{q_{2}}}
$$

Substituting Eq. (2.19) into Eq. (2.14) gives

$$
T(r, \theta)=\sum_{q=-\infty}^{\infty}\left(A_{q_{1}} r^{\beta_{q_{1}}}+A_{q_{2}} r^{\beta_{q_{2}}}\right) \mathrm{e}^{\mathrm{i} q \theta}
$$

The constants $A_{q_{1}}$ and $A_{q_{2}}$ are presented in Appendix.

\section{Solution of the Navier equation}

$$
\begin{array}{ll}
u(r, \theta) & =\sum_{q=-\infty}^{\infty} u_{q}(r) \mathrm{e}^{\left(\mathrm{i} q+n_{1}\right) \theta} \quad v(r, \theta)=\sum_{q=-\infty}^{\infty} v_{q}(r) \mathrm{e}^{\left(\mathrm{i} q+n_{1}\right) \theta} \\
\psi(r, \theta) & =\sum_{q=-\infty}^{\infty} \psi_{q}(r) \mathrm{e}^{\left(\mathrm{i} q+n_{1}\right) \theta}
\end{array}
$$

Substituting Eqs. (2.20) and (3.1) into Eqs. (2.10) yields

$$
\begin{aligned}
u_{q}^{\prime \prime}+ & \zeta_{1} \frac{1}{r} u_{q}^{\prime}+\left(\tau_{2}+\mathrm{i} \tau_{3}\right) \frac{1}{r^{2}} u_{q}+\left(\tau_{4}+\mathrm{i} \tau_{5}\right) \frac{1}{r} v_{q}^{\prime}+\left(\tau_{6}+\mathrm{i} \tau_{7}\right) \frac{1}{r^{2}} v_{q}+\tau_{8} \psi_{q}^{\prime \prime}+\tau_{9} \frac{1}{r} \psi_{q}^{\prime} \\
& +\left(\tau_{10}+\mathrm{i} \tau_{11}\right) \frac{1}{r^{2}} \psi_{q}=\frac{1}{a^{m_{1}}}\left[\left(\tau_{12}+\beta_{q_{1}} \tau_{13}\right) A_{q_{1}} r^{m_{1}+\beta_{q_{1}}-1}+\left(\tau_{12}+\beta_{q_{2}} \tau_{13}\right) A_{q_{2}} r^{m_{1}+\beta_{q_{2}}-1}\right] \\
v_{q}^{\prime \prime} & +\tau_{14} \frac{1}{r} v_{q}^{\prime}-\left(\tau_{15}-\mathrm{i} \tau_{16}\right) \frac{1}{r^{2}} v_{q}+\left(\tau_{17}+\mathrm{i} \tau_{18}\right) \frac{1}{r} u_{q}^{\prime}+\left(\tau_{19}+\mathrm{i} \tau_{20}\right) \frac{1}{r^{2}} u_{q}+\left(\tau_{21}+\mathrm{i} \tau_{22}\right) \frac{1}{r} \psi_{q}^{\prime} \\
& +\left(\tau_{23}+\mathrm{i} \tau_{24}\right) \frac{1}{r^{2}} \psi_{q}=\frac{1}{a^{m_{1}}}\left(\tau_{25}+\mathrm{i} \tau_{26}\right)\left(A_{q_{1}} r^{\beta_{q_{1}}+m_{1}-1}+A_{q_{2}} r^{\beta_{q_{2}}+m_{1}-1}\right) \\
\psi_{q}^{\prime \prime} & +\tau_{27} \frac{1}{r} \psi_{q}^{\prime}+\left(\tau_{28}+\mathrm{i} \tau_{29}\right) \frac{1}{r^{2}} \psi_{q}-\tau_{30} u_{q}^{\prime \prime}-\tau_{31} \frac{1}{r} u_{q}^{\prime}-\tau_{32} \frac{1}{r^{2}} u_{q}+\left(\tau_{33}+\mathrm{i} \tau_{34}\right) \frac{1}{r} v_{q}^{\prime} \\
& +\left(\tau_{35}+\mathrm{i} \tau_{36}\right) \frac{1}{r^{2}} v_{q}=\frac{1}{a^{m_{1}}}\left[\left(\tau_{37}+\mathrm{i} \tau_{38}+\beta_{q_{1}} \tau_{39}\right) A_{q_{1}} r^{\beta_{q_{1}}+m_{1}-1}\right. \\
& \left.+\left(\tau_{37}+\mathrm{i} \tau_{38}+\beta_{q_{2}} \tau_{39}\right) A_{q_{2}} r^{\beta_{q_{2}}+m_{1}-1}\right]
\end{aligned}
$$


Equations (3.2) are a system of ordinary differential equations having general and particular solutions.

The general solutions are assumed as

$$
u_{q}^{g}(r)=D r^{\eta} \quad v_{q}^{g}(r)=E r^{\eta} \quad \psi_{q}^{g}(r)=F r^{\eta}
$$

Substituting Eqs. (3.3) into Eqs. (3.2) yields

$$
\begin{aligned}
& {\left[\eta(\eta-1)+\tau_{1} \eta+\tau_{2}+\mathrm{i} \tau_{3}\right] D+\left[\tau_{4} \eta+\tau_{5}+\mathrm{i}\left(\tau_{6} \eta+\tau_{7}\right)\right] E} \\
& \quad+\left[\eta(\eta-1) \tau_{8}+\tau_{9} \eta+\tau_{10}+\mathrm{i} \tau_{11}\right] F=0 \\
& \quad\left[\tau_{19}+\tau_{17} \eta+\mathrm{i}\left(\tau_{18} \eta+\tau_{20}\right)\right] D+\left[\eta(\eta-1)+\tau_{14} \eta-\tau_{15}+\mathrm{i} \tau_{16}\right] E \\
& \quad+\left[\tau_{21} \eta+\tau_{23}+\mathrm{i}\left(\tau_{22} \eta+\tau_{24}\right)\right] F=0 \\
& \left.\quad \eta(\eta-1) \tau_{30}-\tau_{31} \eta-\tau_{32}\right] D+\left[\tau_{33} \eta+\tau_{35}+\mathrm{i}\left(\tau_{34} \eta+\tau_{36}\right)\right] E \\
& \quad+\left[\eta(\eta-1)+\tau_{27} \eta+\tau_{28}+\mathrm{i} \tau_{29}\right] F=0
\end{aligned}
$$

The constants $\tau_{i}$ are presented in Appendix.

A nontrivial solution is obtained by setting the determinant of the coefficients of Eqs. (3.4) equal to zero, where a six-order polynomial characteristic equation is obtained. It gives six eigenvalues $\eta_{q_{1}}$ to $\eta_{q_{6}}$. Thus, the general solutions are

$$
\begin{array}{llll}
u_{q}^{g}(r)=\sum_{j=1}^{6} D_{q_{j}} r^{\eta_{q_{j}}} & \Rightarrow & u_{q}^{g}(r)=\sum_{j=1}^{6} D_{q_{j}} r^{\eta_{q_{j}}} \\
v_{q}^{g}(r)=\sum_{j=1}^{6} E_{q_{j}} r^{\eta_{q_{j}}} & \Rightarrow & v_{q}^{g}(r)=\sum_{j=1}^{6} X_{q_{j}} D_{q_{j}} r^{\eta_{q_{j}}} \\
\psi_{q}^{g}(r)=\sum_{j=1}^{6} F_{q_{j}} r^{\eta_{q_{j}}} & \Rightarrow & \psi_{q}^{g}(r)=\sum_{j=1}^{6} Y_{q_{j}} D_{q_{j}} r^{\eta_{q_{j}}}
\end{array}
$$

where $X_{q_{j}}$ is the relation between constants $D_{q_{j}}$ and $E_{q_{j}}$ and $Y_{q_{j}}$ is the relation between constants $D_{q_{j}}$ and $F_{q_{j}}$. It is obtained from Eqs. (3.4). The constants are presented in Appendix.

The particular solutions $u_{q}^{p}(r)$ and $v_{q}^{p}(r)$ are assumed as

$$
\begin{aligned}
& u_{q}^{p}(r)=I_{q_{1}} r^{\beta_{q_{1}}+m_{1}+1}+I_{q_{2}} r^{\beta_{q_{2}}+m_{1}+1} \\
& \psi_{q}^{p}(r)=I_{q_{5}} r^{\beta_{q_{1}}+m_{1}+1}+I_{q_{6}} r^{\beta_{q_{2}}+m_{1}+1}
\end{aligned} \quad v_{q}^{p}(r)=I_{q_{3}} r^{\beta_{q_{1}}+m_{1}+1}+I_{q_{4}} r^{\beta_{q_{2}}+m_{1}+1}
$$

Substituting Eqs. (3.6) into the non-homogeneous form of Eqs. (3.2) gives $I_{q_{1}}$ to $I_{q_{6}}$, as they are presented in Appendix. The complete solutions for $u_{q}(r), v_{q}(r)$ and $\psi_{q}(r)$ are the sum of the general and particular solutions

$$
\begin{aligned}
& u_{q}(r)=\sum_{j=1}^{6} D_{q j} r^{\eta_{q j}}+I_{q_{1}} r^{\beta_{q_{1}}+m_{1}+1}+I_{q_{2}} r^{\beta_{q_{2}}+m_{1}+1} \\
& v_{q}(r)=\sum_{j=1}^{6} X_{q j} D_{q j} r^{\eta_{q j}}+I_{q_{3}} r^{\beta_{q_{1}}+m_{1}+1}+I_{q_{4}} r^{\beta_{q_{2}}+m_{1}+1} \\
& \psi_{q}(r)=\sum_{j=1}^{6} Y_{q j} D_{q j} r^{\eta_{q j}}+I_{q_{5}} r^{\beta_{q_{1}}+m_{1}+1}+I_{q_{6}} r^{\beta_{q_{2}}+m_{1}+1}
\end{aligned}
$$


Substituting Eqs. (3.7) into Eqs. (3.1) gives

$$
\begin{aligned}
& u(r, \theta)=\sum_{\substack{q=-\infty \\
q \neq 0}}^{\infty}\left(\sum_{j=1}^{6} D_{q j} r^{\eta_{q j}}+I_{q_{1}} r^{\beta_{q_{1}}+m_{1}+1}+I_{q_{2}} r^{\beta_{q_{2}}+m_{1}+1}\right) \mathrm{e}^{\left(\mathrm{i} q+n_{1}\right) \theta} \\
& v(r, \theta)=\sum_{\substack{q=-\infty \\
q \neq 0}}^{\infty}\left(\sum_{j=1}^{6} X_{q j} D_{q j} r^{\eta_{q j}}+I_{q_{3}} r^{\beta_{q_{1}}+m_{1}+1}+I_{q_{4}} r^{\beta_{q_{2}}+m_{1}+1}\right) \mathrm{e}^{\left(\mathrm{i} q+n_{1}\right) \theta} \\
& \psi(r, \theta)=\sum_{\substack{q=-\infty \\
q \neq 0}}^{\infty}\left(\sum_{j=1}^{6} Y_{q j} D_{q j} r^{\eta_{q j}}+I_{q_{5}} r^{\beta_{q_{1}}+m_{1}+1}+I_{q_{6}} r^{\beta_{q_{2}}+m_{1}+1}\right) \mathrm{e}^{\left(\mathrm{i} q+n_{1}\right) \theta}
\end{aligned}
$$

Substituting Eqs. (3.8) into Eqs. (2.1), the strains and electric intensity are obtained as

$$
\begin{aligned}
\varepsilon_{r r} & =\sum_{\substack{q=-\infty \\
q \neq 0}}^{\infty}\left(\sum_{j=1}^{6} \eta_{q j} D_{q j} r^{\eta_{q j}-1}+\left(\beta_{q_{1}}+m_{1}+1\right) I_{q_{1}} r^{\beta_{q_{1}}+m_{1}}\right. \\
& \left.+\left(\beta_{q_{2}}+m_{1}+1\right) I_{q_{2}} r^{\beta_{q_{2}}+m_{1}}\right) \mathrm{e}^{\left(\mathrm{i} q+n_{1}\right) \theta} \\
\varepsilon_{\theta \theta}= & \sum_{\substack{q=-\infty \\
q \neq 0}}^{\infty}\left(\sum_{j=1}^{6}\left(\mathrm{i} q+n_{1}\right)\left(X_{q j}+1\right) D_{q j} r^{\eta_{q j}-1}+\left[\left(\mathrm{i} q+n_{1}\right) I_{q_{3}}+I_{q_{1}}\right] r^{\beta_{q_{1}}+m_{1}}\right. \\
& \left.+\left[\left(\mathrm{i} q+n_{1}\right) I_{q_{4}}+I_{q_{2}}\right] r^{\beta_{q_{2}}+m_{1}}\right) \mathrm{e}^{\left(\mathrm{i} q+n_{1}\right) \theta} \\
\varepsilon_{r \theta}= & \frac{1}{2} \sum_{\substack{q=-\infty \\
q \neq 0}}^{\infty}\left(\sum_{j=1}^{6}\left[\mathrm{i} q+n_{1}+\left(\eta_{q j}-1\right) X_{q j}\right] D_{q j} r^{\eta_{q j}-1}+\left[\left(\mathrm{i} q+n_{1}\right) I_{q_{1}}\right.\right. \\
& \left.\left.+\left(\beta_{q_{1}}+m_{1}\right) I_{q_{3}}\right] r^{\beta_{q_{1}}+m_{1}}+\left[\left(\mathrm{i} q+n_{1}\right) I_{q_{2}}+\left(\beta_{q_{2}}+m_{1}\right) I_{q_{4}}\right] r^{\beta_{q_{2}}+m_{1}}\right) \mathrm{e}^{\left(\mathrm{i} q+n_{1}\right) \theta} \\
E_{r}= & \sum_{\substack{q=-\infty \\
q \neq 0}}^{\infty}\left(\sum_{j=1}^{6} \eta_{q j} Y_{q j} D_{q j} r^{\eta_{q j}-1}+\left(\beta_{q_{1}}+m_{1}+1\right) I_{q_{5}} r^{\beta_{q_{1}}+m_{1}}\right. \\
& \left.+\left(\beta_{q_{2}}+m_{1}+1\right) I_{q_{6}} r^{\beta_{q_{2}}+m_{1}}\right) \mathrm{e}^{\left(\mathrm{i} q+n_{1}\right) \theta} \\
E_{\theta}= & \sum_{\substack{q=-\infty \\
q \neq 0}}^{\infty}\left(\sum_{j=1}^{6}\left(\mathrm{i} q+n_{1}\right) Y_{q j} D_{q j} r^{\eta_{q j}-1}+\left(\mathrm{i} q+n_{1}\right) I_{q_{5}} r^{\beta_{q_{1}}+m_{1}}+\left(\mathrm{i} q+n_{1}\right) I_{q_{6}} r^{\beta_{q_{2}}+m_{1}}\right) \mathrm{e}^{\left(\mathrm{i} q+n_{1}\right) \theta}
\end{aligned}
$$

Substituting Eqs. (3.9) into Eqs. (2.4), the stresses and electric displacement are obtained as

$$
\begin{aligned}
\sigma_{r r} & =\frac{1}{a^{m_{2}}} \sum_{\substack{q=-\infty \\
q \neq 0}}^{\infty}\left\{\sum _ { j = 1 } ^ { 6 } \left(\widehat { \overline { C } } _ { 1 1 } \left[\eta_{q j} D_{q j} r^{\eta_{q j}+m_{2}-1}+\left(\beta_{q_{1}}+m_{1}+1\right) I_{q_{1}} r^{\beta_{q_{1}}+m_{1}+m_{2}}\right.\right.\right. \\
& \left.+\left(\beta_{q_{2}}+m_{1}+1\right) I_{q_{2}} r^{\beta_{q_{2}}+m_{1}+m_{2}}\right]-\frac{\alpha_{0}}{a^{m_{1}}} \bar{C}_{11}\left(A_{q 1} r^{\beta_{q_{1}}+m_{1}+m_{2}}+A_{q 2} r^{\beta_{q_{2}}+m_{1}+m_{2}}\right) \\
& +\widehat{\bar{C}}_{12}\left[\left(\mathrm{i} q+n_{1}\right)\left(X_{q j}+1\right) D_{q j} r^{\eta_{q j}+m_{2}-1}+\left(\left(\mathrm{i} q+n_{1}\right) I_{q_{3}}+I_{q_{1}}\right) r^{\beta_{q_{1}}+m_{1}+m_{2}}\right. \\
& \left.\left.+\left(\left(\mathrm{i} q+n_{1}\right) I_{q_{4}}+I_{q_{2}}\right) r^{\beta_{q_{2}}+m_{1}+m_{2}}\right]-\frac{2 \alpha_{0}}{a^{m_{1}}} \bar{C}_{12}\left(A_{q 1} r^{\beta_{q_{1}}+m_{1}+m_{2}}+A_{q 2} r^{\beta_{q_{2}}+m_{1}+m_{2}}\right)\right) \mathrm{e}^{n_{2} \theta}
\end{aligned}
$$




$$
\begin{aligned}
& +\bar{e}_{21}\left[\eta_{q j} Y_{q j} D_{q j} r^{\eta_{q j}+m_{2}-1}+\left(\beta_{q_{1}}+m_{1}+1\right) I_{q_{5}} r^{\beta_{q_{1}}+m_{1}+m_{2}}\right. \\
& \left.\left.+\left(\beta_{q_{2}}+m_{1}+1\right) I_{q_{6}} r^{\beta_{q_{2}}+m_{1}+m_{2}}\right] \mathrm{e}^{n_{2} \theta}\right\} \mathrm{e}^{\left(\mathrm{i} q+n_{1}\right) \theta} \\
& \sigma_{\theta \theta}=\frac{1}{a^{m_{2}}} \sum_{\substack{q=-\infty \\
q \neq 0}}^{\infty}\left\{\sum _ { j = 1 } ^ { 6 } \left(\widehat { \overline { C } } _ { 1 2 } \left[\eta_{q j} D_{q j} r^{\eta_{q j}+m_{2}-1}+\left(\beta_{q_{1}}+m_{1}+1\right) I_{q_{1}} r^{\beta_{q_{1}}+m_{1}+m_{2}}\right.\right.\right. \\
& \left.+\left(\beta_{q_{2}}+m_{1}+1\right) I_{q_{2}} r^{\beta_{q_{2}}+m_{1}+m_{2}}\right]-\frac{\alpha_{0}}{a^{m_{1}}} \bar{C}_{12}\left(A_{q 1} r^{\beta_{q_{1}}+m_{1}+m_{2}}+A_{q 2} r^{\beta_{q_{2}}+m_{1}+m_{2}}\right) \\
& +\widehat{\bar{C}}_{22}\left[\left(\mathrm{i} q+n_{1}\right)\left(X_{q j}+1\right) D_{q j} r^{\eta_{q j}+m_{2}-1}+\left(\left(\mathrm{i} q+n_{1}\right) I_{q_{3}}+I_{q_{1}}\right) r^{\beta_{q_{1}}+m_{1}+m_{2}}\right. \\
& \left.\left.+\left(\left(\mathrm{i} q+n_{1}\right) I_{q_{4}}+I_{q_{2}}\right) r^{\beta_{q_{2}}+m_{1}+m_{2}}\right]-\frac{2 \alpha_{0}}{a^{m_{1}}} \bar{C}_{22}\left(A_{q 1} r^{\beta_{q_{1}}+m_{1}+m_{2}}+A_{q 2} r^{\beta_{q_{2}}+m_{1}+m_{2}}\right)\right) \mathrm{e}^{n_{2} \theta} \\
& +\bar{e}_{22}\left[\eta_{q j} Y_{q j} D_{q j} r^{\eta_{q j}+m_{2}-1}+\left(\beta_{q_{1}}+m_{1}+1\right) I_{q_{5}} r^{\beta_{q_{1}}+m_{1}+m_{2}}\right. \\
& \left.\left.+\left(\beta_{q_{2}}+m_{1}+1\right) I_{q_{6}} r^{\beta_{q_{2}}+m_{1}+m_{2}}\right] \mathrm{e}^{n_{2} \theta}\right\} \mathrm{e}^{\left(\mathrm{i} q+n_{1}\right) \theta} \\
& \sigma_{r \theta}=\frac{1}{a^{m_{2}}} \sum_{\substack{q=-\infty \\
q \neq 0}}^{\infty}\left\{\sum _ { j = 1 } ^ { 6 } \overline { C } _ { 4 4 } \left(\left[\left(\mathrm{i} q+n_{1}\right)+\left(\eta_{q j}-1\right) X_{q j}\right] D_{q j} r^{\eta_{q j}+m_{2}-1}+\left[\left(\mathrm{i} q+n_{1}\right) I_{q_{1}}\right.\right.\right. \\
& \left.\left.+\left(\beta_{q_{1}}+m_{1}\right) I_{q_{3}}\right] r^{\beta_{q_{1}}+m_{1}+m_{2}}+\left[\left(\mathrm{i} q+n_{1}\right) I_{q_{2}}+\left(\beta_{q_{2}}+m_{1}\right) I_{q_{4}}\right] r^{\beta_{q_{2}}+m_{1}+m_{2}}\right) \mathrm{e}^{n_{2} \theta} \\
& -\bar{e}_{24}\left[\left(\mathrm{i} q+n_{1}\right) Y_{q j} D_{q j} r^{\eta_{q j}+m_{2}-1}+\left(\mathrm{i} q+n_{1}\right) I_{q_{5}} r^{\beta_{q_{1}}+m_{1}+m_{2}}\right. \\
& \left.\left.+\left(\mathrm{i} q+n_{1}\right) I_{q_{6}} r^{\beta_{q_{2}}+m_{1}+m_{2}}\right] \mathrm{e}^{n_{2} \theta}\right\} \mathrm{e}^{\left(\mathrm{i} q+n_{1}\right) \theta} \\
& \sigma_{z z}=\frac{1}{a^{m_{2}}} \sum_{\substack{q=-\infty \\
q \neq 0}}^{\infty}\left\{\sum _ { j = 1 } ^ { 6 } \left(\widehat { \overline { C } } _ { 1 2 } \left[\eta_{q j} D_{q j} r^{\eta_{q j}+m_{2}-1}+\left(\beta_{q_{1}}+m_{1}+1\right) I_{q_{1}} r^{\beta_{q_{1}}+m_{1}+m_{2}}\right.\right.\right. \\
& +\left(\beta_{q_{2}}+m_{1}+1\right) I_{q_{2}} r^{\beta_{q_{2}}+m_{1}+m_{2}}+\left(\left(\mathrm{i} q+n_{1}\right) I_{q_{3}}+I_{q_{1}}\right) r^{\beta_{q_{1}}+m_{1}+m_{2}} \\
& \left.\left.+\left(\left(\mathrm{i} q+n_{1}\right) I_{q_{4}}+I_{q_{2}}\right) r^{\beta_{q_{2}}+m_{1}+m_{2}}-\frac{3 \alpha_{0}}{a^{m_{1}}} \bar{C}_{12}\left(A_{q 1} r^{\beta_{q_{1}}+m_{1}+m_{2}}+A_{q 2} r^{\beta_{q_{2}}+m_{1}+m_{2}}\right)\right]\right) \mathrm{e}^{n_{2} \theta} \\
& +\bar{e}_{23}\left[\eta_{q j} Y_{q j} D_{q j} r^{\eta_{q j}+m_{2}-1}+\left(\beta_{q_{1}}+m_{1}+1\right) I_{q 5} r^{\beta_{q_{1}}+m_{1}+m_{2}}\right. \\
& \left.\left.+\left(\beta_{q_{2}}+m_{1}+1\right) I_{q_{6}} r^{\beta_{q_{2}}+m_{1}+m_{2}}\right] \mathrm{e}^{n_{2} \theta}\right\} \mathrm{e}^{\left(\mathrm{i} q+n_{1}\right) \theta} \\
& D_{r r}=\frac{1}{a^{m_{2}}} \sum_{\substack{q=-\infty \\
q \neq 0}}^{\infty}\left\{\sum _ { j = 1 } ^ { 6 } \left(\overline { e } _ { 2 1 } \left[\eta_{q j} D_{q j} r^{\eta_{q j}+m_{2}-1}+\left(\beta_{q_{1}}+m_{1}+1\right) I_{q_{1}} r^{\beta_{q_{1}}+m_{1}+m_{2}}\right.\right.\right. \\
& \left.+\left(\beta_{q_{2}}+m_{1}+1\right) I_{q_{2}} r^{\beta_{q_{2}}+m_{1}+m_{2}}\right]+\bar{e}_{22}\left[\left(\mathrm{i} q+n_{1}\right)\left(X_{q j}+1\right) D_{q j} r^{\eta_{q j}+m_{2}-1}\right. \\
& \left.\left.+\left(\left(\mathrm{i} q+n_{1}\right) I_{q_{3}}+I_{q_{1}}\right) r^{\beta_{q_{1}}+m_{1}+m_{2}}+\left(\left(\mathrm{i} q+n_{1}\right) I_{q_{4}}+I_{q_{2}}\right) r^{\beta_{q_{2}}+m_{1}+m_{2}}\right]\right) \mathrm{e}^{n_{2} \theta} \\
& -\bar{\varepsilon}_{22}\left[\eta_{q j} Y_{q j} D_{q j} r^{\eta_{q j}+m_{2}-1}+\left(\beta_{q_{1}}+m_{1}+1\right) I_{q_{5}} r^{\beta_{q_{1}}+m_{1}+m_{2}}\right. \\
& \left.+\left(\beta_{q_{2}}+m_{1}+1\right) I_{q_{6}} r^{\beta_{q_{2}}+m_{1}+m_{2}}\right] \mathrm{e}^{n_{2} \theta} \\
& \left.+\frac{\bar{g}_{21}}{a^{m_{1}}}\left(A_{q_{1}} r^{\beta_{q_{1}}+m_{1}+m_{2}}+A_{q_{2}} r^{\beta_{q_{2}}+m_{1}+m_{2}}\right) \mathrm{e}^{\left(n_{1}+n_{2}\right) \theta}\right\} \mathrm{e}^{\left(\mathrm{i} q+n_{1}\right) \theta}
\end{aligned}
$$




$$
\begin{aligned}
D_{\theta \theta} & =\frac{1}{a^{m_{2}}} \sum_{\substack{q=-\infty \\
q \neq 0}}^{\infty}\left\{\sum _ { j = 1 } ^ { 6 } \overline { e } _ { 2 4 } \left(\left[\mathrm{i} q+n_{1}+\left(\eta_{q j}-1\right) X_{q j}\right] D_{q j} r^{\eta_{q j}+m_{4}-1}+\left[\left(\mathrm{i} q+n_{1}\right) I_{q_{1}}\right.\right.\right. \\
& \left.\left.+\left(\beta_{q_{1}}+m_{1}\right) I_{q_{3}}\right] r^{\beta_{q_{1}}+m_{1}+m_{4}}+\left[\left(\mathrm{i} q+n_{1}\right) I_{q_{2}}+\left(\beta_{q_{2}}+m_{1}\right) I_{q_{4}}\right] r^{\beta_{q_{2}}+m_{1}+m_{4}}\right) \mathrm{e}^{n_{4} \theta} \\
& -\bar{\varepsilon}_{21}\left(\left(\mathrm{i} q+n_{1}\right) Y_{q j} D_{q j} r^{\eta_{q j}+m_{5}-1}+\left(\mathrm{i} q+n_{1}\right) I_{q_{5}} r^{\beta_{q_{1}}+m_{1}+m_{5}}\right. \\
& \left.\left.+\left(\mathrm{i} q+n_{1}\right) I_{q_{6}} r^{\beta_{q_{2}}+m_{1}+m_{5}}\right) \mathrm{e}^{n_{5} \theta}+\frac{\bar{g}_{22}}{a^{m_{1}}}\left(A_{q_{1}} r^{\beta_{q_{1}}+m_{1}+m_{6}}+A_{q_{2}} r^{\beta_{q_{2}}+m_{1}+m_{6}}\right) \mathrm{e}^{n_{6} \theta}\right\} \mathrm{e}^{\left(\mathrm{i} q+n_{1}\right) \theta}
\end{aligned}
$$

To determine the constants $D_{q j}$, any general from of boundary conditions for displacements, stresses and potential electric is considered as

$$
\begin{array}{lll}
u(a, \theta)=w_{1}(\theta) & u(b, \theta)=w_{2}(\theta) & v(a, \theta)=w_{3}(\theta) \\
v(b, \theta)=w_{4}(\theta) & \sigma_{r r}(a, \theta)=w_{7}(\theta) & \sigma_{r r}(b, \theta)=w_{8}(\theta) \\
\sigma_{r \theta}(a, \theta)=w_{9}(\theta) & \sigma_{r \theta}(b, \theta)=w_{10}(\theta) & \psi(a, \theta)=w_{5}(\theta) \\
\psi(b, \theta)=w_{6}(\theta) & D_{r r}(a, \theta)=w_{11}(\theta) & D_{r r}(b, \theta)=w_{12}(\theta)
\end{array}
$$

It is recalled that Eqs. (3.9) and (3.10) contain six unknowns, $D_{q_{1}}, D_{q_{2}}, \ldots, D_{q_{6}}$. Assume that the six boundary conditions are specified from list of Eqs. (3.11). The boundary conditions may be either the given displacements and electric potential or stresses, or combinations. Expanding the given boundary conditions in complex Fourier series gives

$$
w_{j}(\theta)=\sum_{n=-\infty}^{\infty} W_{j}(q) \mathrm{e}^{\left(\mathrm{i} q+n_{1}\right) \theta} \quad j=1, \ldots, 6
$$

where

$$
W_{j}(q)=\frac{1}{2 \pi} \int_{-\pi}^{\pi} w_{j}(q) \mathrm{e}^{-\left(\mathrm{i} q+n_{1}\right) \theta} d \theta \quad j=1, \ldots, 6
$$

Using the selected six boundary conditions of Eqs. (3.11) with the help of Eqs. (3.12) and (3.13), the six unknown coefficients $D_{q_{1}}$ to $D_{q_{6}}$ are calculated.

\section{Results and discussion}

Consider a thick hollow cylinder of inner radius $a=1 \mathrm{~m}$ and outer radius $b=1.2 \mathrm{~m}$ of $\mathrm{Ba}_{2} \mathrm{NaNb}_{5} \mathrm{O}_{15}$ material with properties given in Table 1.

The thermal boundary conditions are substituted into Eq. (2.12) to obtain the temperature distribution, where the constants of integration are obtained from the equations given in Appendix. The stress and displacement and electric potential boundary conditions are assumed to be selected such that the mathematical strength of the proposed method can be examined. These type of boundary conditions may not be handled with the potential function method. The constant coefficients of the series expansions are obtained from Eq. (3.13). Here, $B$ is the compressibility coefficient, sometimes named the skempton pore pressure coefficient, and $\phi$ is the pore volume fraction and is pore per total volume, respectively, which are given in Appendix. Using Eqs. (3.11) and (3.12), the boundary conditions given in terms of the radial and shear stresses as well as electric potential appear in Table 2 . These boundary conditions are expanded by the integral series and the unknown coefficients $D_{q_{j}}$ are determined. 
Table 1. Material constants of $\mathrm{Ba}_{2} \mathrm{NaNb}_{5} \mathrm{O}_{15}$ for 2D-PPM (Akbari Alashti et al., 2013; Jabbari et al., 2012)

\begin{tabular}{|c|c||c|c||c|c|}
\hline Parameter & Value & Parameter & Value & Parameter & Value \\
\hline \hline$\alpha_{0}\left[1 /{ }^{\circ} \mathrm{C}\right]$ & $1.2 \cdot 10^{-6}$ & $\bar{C}_{11}[\mathrm{GPa}]$ & 239 & $\bar{e}_{22}\left[\mathrm{C} / \mathrm{m}^{2}\right]$ & -0.3 \\
\hline$\gamma$ & 0.75 & $\bar{C}_{12}[\mathrm{GPa}]$ & 104 & $\bar{e}_{24}\left[\mathrm{C} / \mathrm{m}^{2}\right]$ & 3.4 \\
\hline$\nu$ & 0.25 & $\bar{C}_{22}[\mathrm{GPa}]$ & 247 & $\bar{\varepsilon}_{21}\left[\mathrm{C}^{2} / \mathrm{N} \mathrm{m}^{2}\right]$ & $1.96 \cdot 10^{-9}$ \\
\hline$\nu_{u}$ & 0.3 & $\bar{C}_{44}[\mathrm{GPa}]$ & 76 & $\bar{\varepsilon}_{22}\left[\mathrm{C}^{2} / \mathrm{N} \mathrm{m}^{2}\right]$ & $2.01 \cdot 10^{-9}$ \\
\hline$k_{0}[\mathrm{~W} / \mathrm{m} \mathrm{K}]$ & 13.9 & $\bar{e}_{21}\left[\mathrm{C} / \mathrm{m}^{2}\right]$ & -0.4 & $\bar{g}_{21}\left[\mathrm{C} / \mathrm{m}^{2} \mathrm{~K}\right]$ & $5.4 \cdot 10^{-5}$ \\
\hline$m_{1}, m_{2}, \ldots, m_{6}$ & $m$ & $n_{1}, n_{2}, \ldots, n_{6}$ & $n$ & $\bar{g}_{22}\left[\mathrm{C} / \mathrm{m}^{2} \mathrm{~K}\right]$ & $5.4 \cdot 10^{-5}$ \\
\hline
\end{tabular}

Table 2. Boundary condition for 2D-PPM (Jabbari et al., 2012)

\begin{tabular}{|c|c|c|c|c|c|c|}
\hline $\begin{array}{c}T(a, \theta) \\
{\left[{ }^{\circ} \mathrm{C}\right]}\end{array}$ & $T(b, \theta)$ & $\begin{array}{c}\sigma_{r r}(a, \theta) \\
{[\mathrm{MPa}]}\end{array}$ & $\begin{array}{c}\sigma_{r \theta}(a, \theta) \\
{[\mathrm{MPa}]}\end{array}$ & $u(b, \theta)$ & $v(b, \theta)$ & $\begin{array}{c}\psi(a, \theta) \\
{[\mathrm{W} / \mathrm{A}]}\end{array}$ \\
\hline \hline $60 \sin (2|\theta|)$ & 0 & $400 \sin \left(\frac{\theta^{2}}{4}-|\theta|\right)$ & $50 \theta^{2} \cos \theta$ & 0 & 0 & $\psi_{0} \theta^{2} \cos (2 \theta)$ \\
\hline
\end{tabular}

(a)

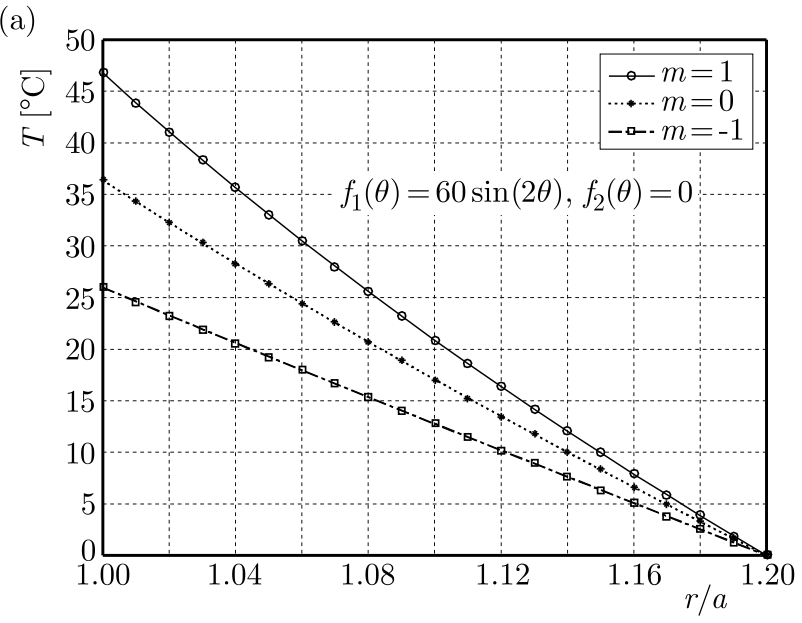

(b)

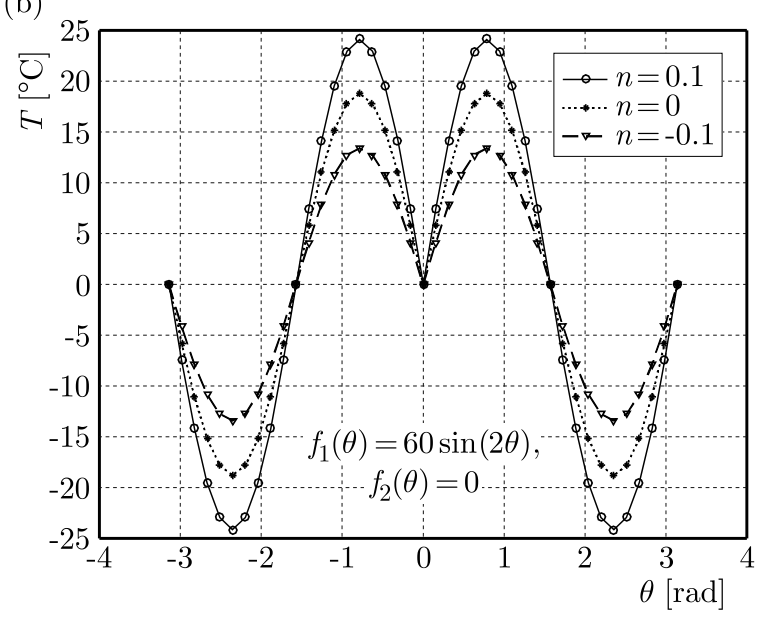

Fig. 2. Temperature distribution in the (a) radial at $\theta=\pi / 3$ and (b) circumferential direction at $r=\bar{r}$
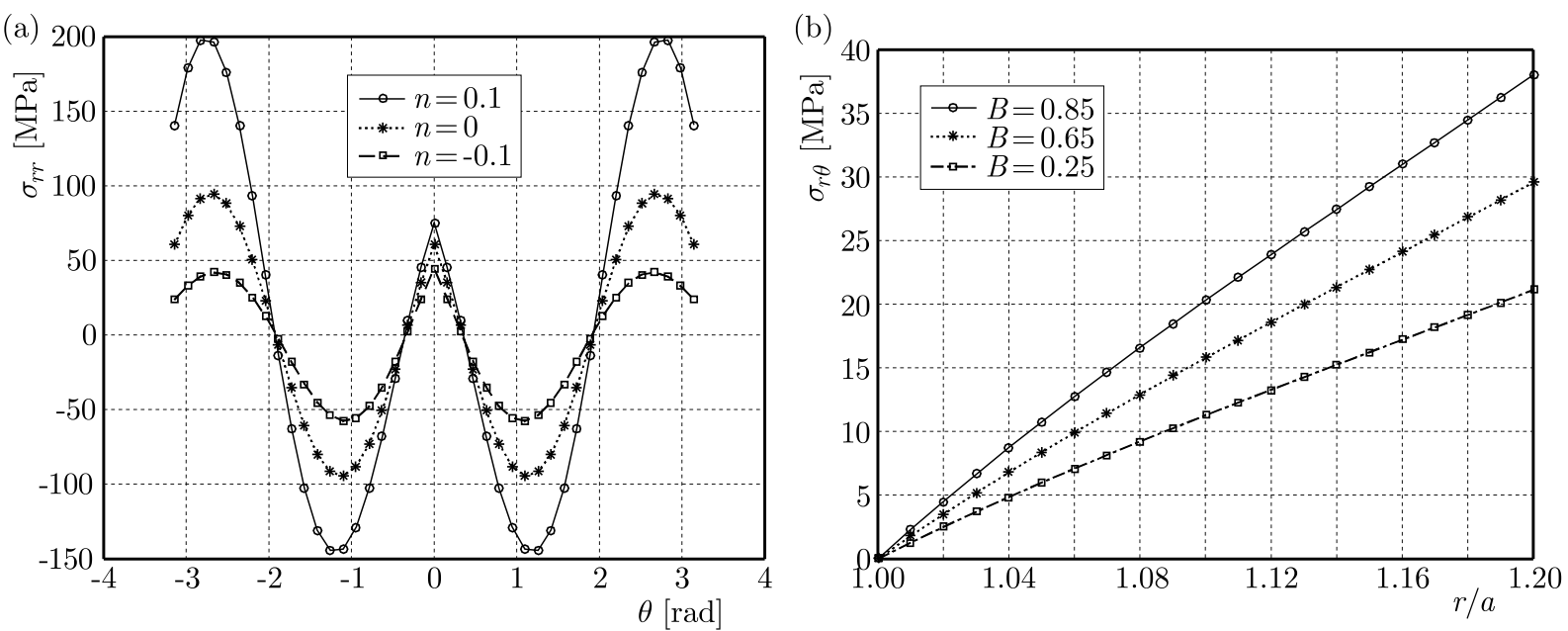

Fig. 3. (a) Circumferential distribution of radial thermo-electro-mechanicalal stresses $\sigma_{r r}$ at $r=\bar{r}$. (b) Radial distribution of shear thermo-electro-mechanicalal stresses $\sigma_{r \theta}$ at $\theta=\pi / 3$ 
Figure $2 \mathrm{a}$ and $2 \mathrm{~b}$ shows the effect of the power-exponential law index on the temperature distribution in the wall thickness along the radial and circumferential directions. The effect of the power-exponential law index on the distribution of the radial thermo-electro-mechanical stresses is shown in Fig. 3a. It is shown that as $m, n$ increases, the radial, hoop, shear and axial thermal stresses are increased. This figure is a plot of stresses versus $\theta$ at $r=\bar{r}=1.1$, where $\bar{r}$ is the avrege inner radius $a$ and the outer radius $b$. Figure $3 \mathrm{~b}$ shows the shear thermo-electro-mechanical stresses in the cross section of the cylinder, where the pore compressibility coefficient $B$ is changed and the other parameters are fixed. Figure 4a shows the radial displacement in the cross section of the cylinder, where the based on the pore volume fraction $\phi$ is changing. Also the electric potential constant in Figs. 1 to $4 \mathrm{a}$ is $\psi_{0}=60 \mathrm{~V}$. Figure $4 \mathrm{~b}$ shows the circumferential displacements in the cross section of the cylinder, where the based on the versus electric potential coefficient $\psi_{0}$ is changing.
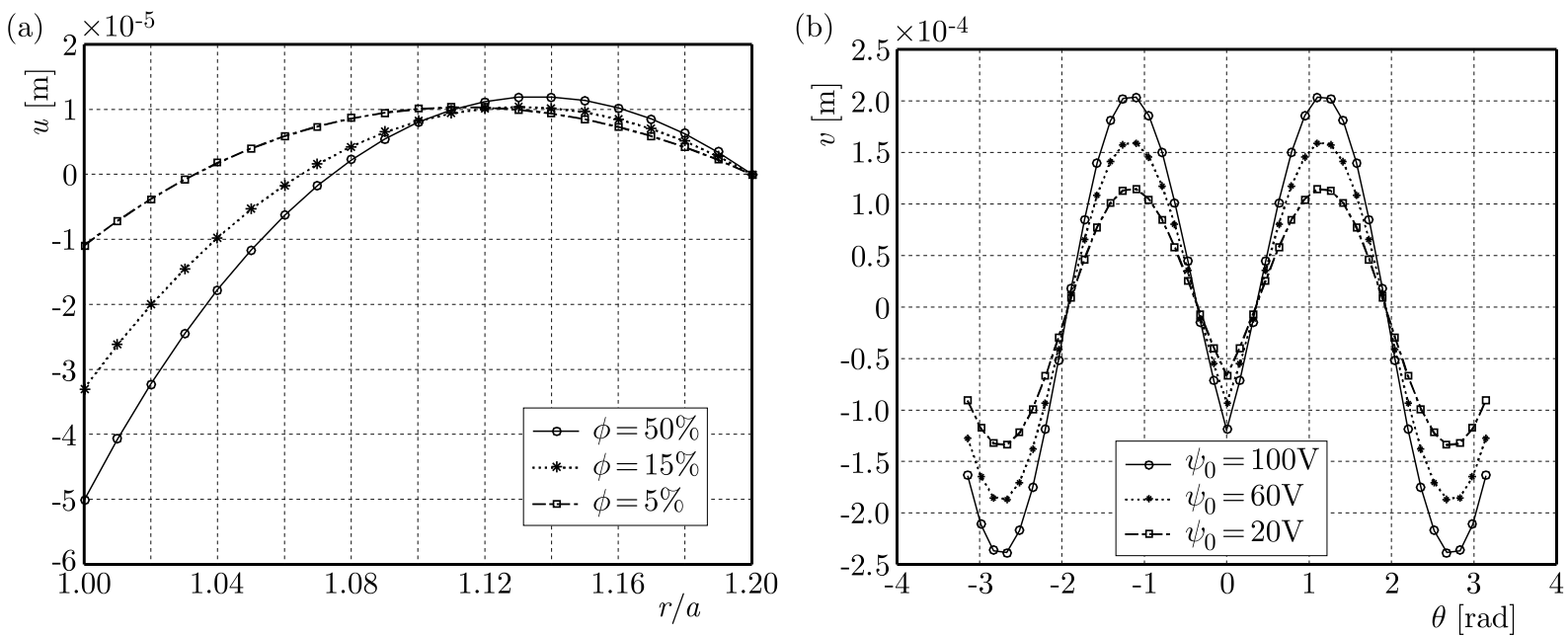

Fig. 4. (a) Radial distribution of $u$ with diffrent porosity cofficient at $\theta=\pi / 3$. (b) Circumferential distribution of $v$ with electric potential coefficient at $r=\bar{r}$

\section{Conclusions}

In the present work, an attempt is made to study the problem of analitical solution for the Thermo-Electro-Mechanical (TEM) in a thick 2D-PPM hollow infinite cylinder where the twodimensional asymmetric steady-state loads are implied. The method of solution is based on the direct method and uses the power series, rather than the potential function method. The advantage of this method is its mathematical power to handle both simple and complicated mathematical functions for the thermal and mechanical stresses boundary conditions. The potential function method is capable of handling complicated mathematical functions as the boundary conditions. The proposed method does not have mathematical limitations to deal with general types of boundary conditions, which usually occur in the potential function method.

\section{Appendix}

$$
\begin{aligned}
d_{1}= & \left(\beta_{q_{1}}+m_{1}+1\right)\left(\beta_{q_{1}}+m_{1}\right)+\left(\left(m_{2}+1\right)+\left(m_{2}-1\right) \frac{\widehat{\bar{C}}_{12}}{\widehat{\bar{C}}_{11}}\right)\left(\beta_{q_{1}}+m_{1}+1\right) \\
& +\frac{m_{2} \widehat{\bar{C}}_{12}-\widehat{\bar{C}}_{22}}{\widehat{\bar{C}}_{11}}+\left[\left(n_{1}+n_{2}\right) n_{1}+\mathrm{i} q\left(2 n_{1}+n_{2}\right)-q^{2}\right] \frac{\bar{C}_{44}}{\widehat{\widehat{C}}_{11}}
\end{aligned}
$$




$$
\begin{aligned}
& d_{2}=\left(\beta_{q_{2}}+m_{1}+1\right)\left(\beta_{q_{2}}+m_{1}\right)+\left(\left(m_{2}+1\right)+\left(m_{2}-1\right) \frac{\widehat{\bar{C}}_{12}}{\widehat{\bar{C}}_{11}}\right)\left(\beta_{q_{2}}+m_{1}+1\right) \\
& +\frac{m_{2} \widehat{\bar{C}}_{12}-\widehat{\bar{C}}_{22}}{\widehat{\bar{C}}_{11}}+\left[\left(n_{1}+n_{2}\right) n_{1}+\mathrm{i} q\left(2 n_{1}+n_{2}\right)-q^{2}\right] \frac{\bar{C}_{44}}{\widehat{\bar{C}}_{11}} \\
& d_{3}=\left(\left(\mathrm{i} q+n_{1}\right) \frac{\widehat{\bar{C}}_{12}}{\widehat{\bar{C}}_{11}}+\left(\mathrm{i} q+n_{1}+n_{2}\right) \frac{\bar{C}_{44}}{\widehat{\bar{C}}_{11}}\right)\left(\beta_{q_{1}}+m_{1}+1\right) \\
& +\left(\mathrm{i} q+n_{1}\right) \frac{m_{2} \widehat{\bar{C}}_{12}-\widehat{\bar{C}}_{22}}{\widehat{\bar{C}}_{11}}-\left(\mathrm{i} q+n_{1}+n_{2}\right) \frac{\bar{C}_{44}}{\hat{\bar{C}}_{11}} \\
& d_{4}=\left(\left(\mathrm{i} q+n_{1}\right) \frac{\widehat{\bar{C}}_{12}}{\widehat{\bar{C}}_{11}}+\left(\mathrm{i} q+n_{1}+n_{2}\right) \frac{\bar{C}_{44}}{\widehat{\bar{C}}_{11}}\right)\left(\beta_{q_{2}}+m_{1}+1\right) \\
& +\left(\mathrm{i} q+n_{1}\right) \frac{m_{2} \widehat{\bar{C}}_{12}-\widehat{\bar{C}}_{22}}{\widehat{\bar{C}}_{11}}-\left(\mathrm{i} q+n_{1}+n_{2}\right) \frac{\bar{C}_{44}}{\hat{\bar{C}}_{11}} \\
& d_{5}=\frac{\bar{e}_{21}}{\widehat{\bar{C}}_{11}}\left(\beta_{q_{1}}+m_{1}+1\right)\left(\beta_{q_{1}}+m_{1}\right)+\frac{\left(m_{4}+1\right) \bar{e}_{21}-\bar{e}_{22}}{\widehat{\bar{C}}_{11}}\left(\beta_{q_{1}}+m_{1}+1\right) \\
& +\left[\left(n_{1}+n_{4}\right) n_{1}+\mathrm{i} q\left(2 n_{1}+n_{4}\right)-q^{2}\right] \frac{\bar{e}_{24}}{\overline{\bar{C}}_{11}} \\
& d_{6}=\frac{\bar{e}_{21}}{\widehat{\bar{C}}_{11}}\left(\beta_{q_{2}}+m_{1}+1\right)\left(\beta_{q_{2}}+m_{1}\right)+\frac{\left(m_{4}+1\right) \bar{e}_{21}-\bar{e}_{22}}{\overline{\bar{C}}_{11}}\left(\beta_{q_{2}}+m_{1}+1\right) \\
& +\left[\left(n_{1}+n_{4}\right) n_{1}+\mathrm{i} q\left(2 n_{1}+n_{4}\right)-q^{2}\right] \frac{\bar{e}_{24}}{\overline{\bar{C}}_{11}} \\
& d_{7}=\left(\frac{\left(m_{1}+m_{2}+1\right) \bar{C}_{11}+2\left(m_{1}+m_{2}\right) \bar{C}_{12}-\bar{C}_{22}}{\widehat{\bar{C}}_{11}}+\frac{\bar{C}_{11}+2 \bar{C}_{12}}{\widehat{\bar{C}}_{11}} \beta_{q_{1}}\right) \frac{\alpha_{0}}{a^{m_{1}}} A_{q_{1}} \\
& d_{8}=\left(\frac{\left(m_{1}+m_{2}+1\right) \bar{C}_{11}+2\left(m_{1}+m_{2}\right) \bar{C}_{12}-\bar{C}_{22}}{\widehat{\bar{C}}_{11}}+\frac{\bar{C}_{11}+2 \bar{C}_{12}}{\widehat{C}_{11}} \beta_{q_{2}}\right) \frac{\alpha_{0}}{a^{m_{1}}} A_{q_{2}} \\
& d_{9}=\left(\beta_{q_{1}}+m_{1}+1\right)\left(\beta_{q_{1}}+m_{1}\right)+\left(m_{2}+1\right)\left(\beta_{q_{1}}+m_{1}+1\right) \\
& \left.-\left(m_{2}+1\right)+\left[\left(n_{1}+n_{2}\right)+\mathrm{i} q\left(n_{2}+2\right)-q^{2}\right] \frac{\widehat{\bar{C}}_{22}}{\bar{C}_{44}}\right) \\
& d_{10}=\left(\beta_{q_{2}}+m_{1}+1\right)\left(\beta_{q_{2}}+m_{1}\right)+\left(m_{2}+1\right)\left(\beta_{q_{2}}+m_{1}+1\right) \\
& -\left(m_{2}+1\right)+\left[\left(n_{1}+n_{2}\right)+\mathrm{i} q\left(n_{2}+2\right)-q^{2}\right] \frac{\widehat{\bar{C}}_{22}}{\bar{C}_{44}} \\
& d_{11}=\left(\left(\mathrm{i} q+n_{1}\right)+\left(\mathrm{i} q+n_{1}+n_{2}\right) \frac{\widehat{\bar{C}}_{12}}{\bar{C}_{44}}\right)\left(\beta_{q_{1}}+m_{1}+1\right) \\
& +\left(\mathrm{i} q+n_{1}\right)\left(m_{2}+1\right)+\left(\mathrm{i} q+n_{1}+n_{2}\right) \frac{\widehat{\bar{C}}_{22}}{\bar{C}_{44}} \\
& d_{12}=\left(\left(\mathrm{i} q+n_{1}\right)+\left(\mathrm{i} q+n_{1}+n_{2}\right) \frac{\widehat{\bar{C}}_{12}}{\bar{C}_{44}}\right)\left(\beta_{q_{2}}+m_{1}+1\right) \\
& +\left(\mathrm{i} q+n_{1}\right)\left(m_{2}+1\right)+\left(\mathrm{i} q+n_{1}+n_{2}\right) \frac{\widehat{\bar{C}}_{22}}{\bar{C}_{44}} \\
& d_{13}=\left(\left(\mathrm{i} q+n_{1}\right) \frac{\bar{e}_{24}}{\bar{C}_{44}}+\left(\mathrm{i} q+n_{1}+n_{4}\right) \frac{\bar{e}_{22}}{\bar{C}_{44}}\right)\left(\beta_{q_{1}}+m_{1}+1\right)+\left(\mathrm{i} q+n_{1}\right)\left(m_{4}+2\right) \frac{\bar{e}_{24}}{\bar{C}_{44}}
\end{aligned}
$$




$$
\begin{aligned}
& d_{14}=\left(\left(\mathrm{i} q+n_{1}\right) \frac{\bar{e}_{24}}{\bar{C}_{44}}+\left(\mathrm{i} q+n_{1}+n_{4}\right) \frac{\bar{e}_{22}}{\bar{C}_{44}}\right)\left(\beta_{q_{2}}+m_{1}+1\right)+\left(\mathrm{i} q+n_{1}\right)\left(m_{4}+2\right) \frac{\bar{e}_{24}}{\bar{C}_{44}} \\
& d_{15}=\left(n_{1}+n_{2}+\mathrm{i} q\right) \frac{2 \bar{C}_{12}+\bar{C}_{22}}{\bar{C}_{44}} \frac{\alpha_{0}}{a^{m_{1}}} A_{q_{1}} \\
& d_{16}=\left(n_{1}+n_{2}+\mathrm{i} q\right) \frac{2 \bar{C}_{12}+\bar{C}_{22}}{\bar{C}_{44}} \frac{\alpha_{0}}{a^{m_{1}}} A_{q_{2}} \\
& d_{17}=\left(\beta_{q_{1}}+m_{1}+1\right)\left(\beta_{q_{1}}+m_{1}\right)+\left(\beta_{q_{1}}+m_{1}+1\right)\left(m_{5}+1\right) \\
& +\left[\left(n_{1}+n_{5}\right) n_{1}+\mathrm{i} q\left(n_{5}+2 n_{1}\right)-q^{2}\right] \frac{\bar{\varepsilon}_{21}}{\bar{\varepsilon}_{22}} \\
& d_{18}=\left(\beta_{q_{2}}+m_{1}+1\right)\left(\beta_{q_{2}}+m_{1}\right)+\left(\beta_{q_{2}}+m_{1}+1\right)\left(m_{5}+1\right) \\
& +\left[\left(n_{1}+n_{5}\right) n_{1}+\mathrm{i} q\left(n_{5}+2 n_{1}\right)-q^{2}\right] \frac{\bar{\varepsilon}_{21}}{\bar{\varepsilon}_{22}} \\
& d_{19}=-\frac{\bar{e}_{21}}{\bar{\varepsilon}_{22}}\left(\beta_{q_{1}}+m_{1}+1\right)\left(\beta_{q_{1}}+m_{1}\right)-\frac{\left(m_{4}+1\right) \bar{e}_{21}+\bar{e}_{22}}{\bar{\varepsilon}_{22}}\left(\beta_{q_{1}}+m_{1}+1\right)-\frac{m_{4} \bar{e}_{22}}{\bar{\varepsilon}_{22}} \\
& d_{20}=-\frac{\bar{e}_{21}}{\bar{\varepsilon}_{22}}\left(\beta_{q_{2}}+m_{1}+1\right)\left(\beta_{q_{2}}+m_{1}\right)-\left(\frac{\left(m_{4}+1\right) \bar{e}_{21}+\bar{e}_{22}}{\bar{\varepsilon}_{22}}\left(\beta_{q_{2}}+m_{1}+1\right)-\frac{m_{4} \bar{e}_{22}}{\bar{\varepsilon}_{22}}\right. \\
& d_{21}=\left(\mathrm{i} q+n_{1}+n_{4}\right) \frac{\bar{e}_{24}}{\bar{\varepsilon}_{22}}\left(\beta_{q_{1}}+m_{1}+1\right)+\left(\mathrm{i} q+n_{1}\right)\left(m_{4}+1\right) \frac{\bar{e}_{22}}{\bar{\varepsilon}_{22}}-\left(\mathrm{i} q+n_{1}+n_{4}\right) \frac{\bar{e}_{24}}{\bar{\varepsilon}_{22}} \\
& d_{22}=\left(\mathrm{i} q+n_{1}+n_{4}\right) \frac{\bar{e}_{24}}{\bar{\varepsilon}_{22}}\left(\beta_{q_{2}}+m_{1}+1\right)+\left(\mathrm{i} q+n_{1}\right)\left(m_{4}+1\right) \frac{\bar{e}_{22}}{\bar{\varepsilon}_{22}}-\left(\mathrm{i} q+n_{1}+n_{4}\right) \frac{\bar{e}_{24}}{\bar{\varepsilon}_{22}} \\
& d_{23}=\left(\left(m_{6}+1\right) \frac{\bar{g}_{21}}{\bar{\varepsilon}_{22}}+\left(\mathrm{i} q+n_{6}+\beta_{q_{1}}\right) \frac{\bar{g}_{22}}{\bar{\varepsilon}_{22}}\right) \frac{1}{a^{m_{1}}} A_{q_{1}} \\
& d_{24}=\left(\left(m_{6}+1\right) \frac{\bar{g}_{21}}{\bar{\varepsilon}_{22}}+\left(\mathrm{i} q+n_{6}+\beta_{q_{2}}\right) \frac{\bar{g}_{22}}{\bar{\varepsilon}_{22}}\right) \frac{1}{a^{m_{1}}} A_{q_{2}} \\
& \widehat{N}_{l q_{j}}=\eta_{q_{j}}\left(\eta_{q_{j}}-1\right)+\tau_{1} \eta+\tau_{2}+\tau_{3}+\mathrm{i} \tau_{4} \\
& \widehat{N}_{2 q_{j}}=\tau_{5} \eta_{q_{j}}+\tau_{7}+\mathrm{i}\left(\tau_{6}+\tau_{8} \eta_{q_{j}}\right) \\
& \widehat{N}_{3 q_{j}}=\eta_{q_{j}}\left(\eta_{q_{j}}-1\right) \tau_{9}+\tau_{10} \eta_{q_{j}}+\tau_{11}+\mathrm{i} \tau_{12} \\
& \widehat{N}_{4 q_{j}}=\tau_{16}+\tau_{18} \eta_{q_{j}}+\mathrm{i}\left(\tau_{19} \eta_{q_{j}}+\tau_{21}\right) \\
& \widehat{N}_{5 q_{j}}=\eta_{q_{j}}\left(\eta_{q_{j}}-1\right)+\tau_{15} \eta_{q_{j}}-\tau_{16}+\mathrm{i} \tau_{17} \\
& \widehat{N}_{6 q_{j}}=\tau_{26} \eta_{q_{j}}+\tau_{28}+\mathrm{i}\left(\tau_{27} \eta_{q_{j}}+\tau_{29}\right) \\
& \widehat{N}_{7 q_{j}}=\eta_{q_{j}}\left(\eta_{q_{j}}-1\right) \tau_{35}-\tau_{36} \eta_{q_{j}}-\tau_{37} \\
& \widehat{N}_{8 q_{j}}=\tau_{38} \eta_{q_{j}}+\tau_{40}+\mathrm{i}\left(\tau_{39} \eta_{q_{j}}+\tau_{41}\right) \\
& \widehat{N}_{9 q_{j}}=\eta_{q_{j}}\left(\eta_{q_{j}}-1\right)-\tau_{32} \eta_{q_{j}}+\tau_{33}+\mathrm{i} \tau_{34} \\
& {\left[\begin{array}{lll}
\widehat{N}_{1 q_{j}} & \widehat{N}_{2 q_{j}} & \widehat{N}_{3 q_{j}} \\
\widehat{N}_{4 q_{j}} & \widehat{N}_{5 q_{j}} & \widehat{N}_{6 q_{j}} \\
\widehat{N}_{7 q_{j}} & \widehat{N}_{8 q_{j}} & \widehat{N}_{9 q_{j}}
\end{array}\right]\left[\begin{array}{c}
D_{q_{j}} \\
E_{q_{j}} \\
F_{q_{j}}
\end{array}\right]=\left[\begin{array}{l}
0 \\
0 \\
0
\end{array}\right] \Rightarrow\left|\begin{array}{lll}
\widehat{N}_{1 q_{j}} & \widehat{N}_{2 q_{j}} & \widehat{N}_{3 q_{j}} \\
\widehat{N}_{4 q_{j}} & \widehat{N}_{5 q_{j}} & \widehat{N}_{6 q_{j}} \\
\widehat{N}_{7 q_{j}} & \widehat{N}_{8 q_{j}} & \widehat{N}_{9 q_{j}}
\end{array}\right|=0} \\
& X_{q j}=\frac{E_{q_{j}}}{D_{q_{j}}}=\frac{\widehat{N}_{1 q_{j}} \widehat{N}_{6 q_{j}}-\widehat{N}_{3 q_{j}} \widehat{N}_{4 q_{j}}}{\widehat{N}_{3 q_{j}} \widehat{N}_{5 q_{j}}-\widehat{N}_{2 q_{j}} \widehat{N}_{6 q_{j}}} \quad Y_{q j}=\frac{F_{q_{j}}}{D_{q_{j}}}=\frac{\widehat{N}_{4 q_{j}} \widehat{N}_{8 q_{j}}-\widehat{N}_{5 q_{j}} \widehat{N}_{7 q_{j}}}{\widehat{N}_{5 q_{j}} \widehat{N}_{9 q_{j}}-\widehat{N}_{6 q_{j}} \widehat{N}_{8 q_{j}}} \quad j=1, \ldots, 6 \\
& I_{q_{1}}=\frac{d_{7} d_{9} d_{17}-d_{3} d_{15} d_{17}-d_{5} d_{9} d_{23}-d_{7} d_{13} d_{19}+d_{3} d_{13} d_{23}+d_{5} d_{15} d_{19}}{d_{1} d_{9} d_{17}-d_{3} d_{11} d_{17}-d_{1} d_{13} d_{19}-d_{5} d_{9} d_{21}+d_{5} d_{11} d_{19}+d_{3} d_{13} d_{21}} \\
& I_{q_{2}}=\frac{d_{8} d_{10} d_{18}-d_{4} d_{16} d_{18}-d_{6} d_{10} d_{24}-d_{8} d_{14} d_{20}+d_{4} d_{14} d_{24}+d_{6} d_{16} d_{20}}{d_{2} d_{10} d_{18}-d_{4} d_{12} d_{18}-d_{2} d_{14} d_{20}-d_{6} d_{10} d_{22}+d_{6} d_{12} d_{20}+d_{4} d_{14} d_{22}} \\
& I_{q_{3}}=\frac{d_{1} d_{15} d_{17}-d_{7} d_{11} d_{17}-d_{1} d_{13} d_{23}-d_{5} d_{15} d_{21}+d_{5} d_{11} d_{23}+d_{7} d_{13} d_{21}}{d_{1} d_{9} d_{17}-d_{3} d_{11} d_{17}-d_{1} d_{13} d_{19}-d_{5} d_{9} d_{21}+d_{5} d_{11} d_{19}+d_{3} d_{13} d_{21}} \\
& I_{q_{4}}=\frac{d_{2} d_{16} d_{18}-d_{8} d_{12} d_{18}-d_{2} d_{14} d_{24}-d_{6} d_{16} d_{22}+d_{6} d_{12} d_{24}+d_{8} d_{14} d_{22}}{d_{2} d_{10} d_{18}-d_{4} d_{12} d_{18}-d_{2} d_{14} d_{20}-d_{6} d_{10} d_{22}+d_{6} d_{12} d_{20}+d_{4} d_{14} d_{22}} \\
& I_{q_{5}}=\frac{d_{1} d_{9} d_{23}-d_{1} d_{15} d_{19}-d_{3} d_{11} d_{23}-d_{7} d_{9} d_{21}+d_{7} d_{11} d_{19}+d_{3} d_{15} d_{21}}{d_{1} d_{9} d_{17}-d_{3} d_{11} d_{17}-d_{1} d_{13} d_{19}-d_{5} d_{9} d_{21}+d_{5} d_{11} d_{19}+d_{3} d_{13} d_{21}} \\
& I_{q_{6}}=\frac{d_{2} d_{10} d_{24}-d_{2} d_{16} d_{20}-d_{4} d_{12} d_{24}-d_{8} d_{10} d_{22}+d_{8} d_{12} d_{20}+d_{4} d_{16} d_{22}}{d_{2} d_{10} d_{18}-d_{4} d_{12} d_{18}-d_{2} d_{14} d_{20}-d_{6} d_{10} d_{22}+d_{6} d_{12} d_{20}+d_{4} d_{14} d_{22}}
\end{aligned}
$$




$$
\begin{aligned}
& \tau_{1}=\left(m_{2}+1\right)+\left(m_{2}-1\right) \frac{\widehat{\bar{C}}_{12}}{\widehat{\bar{C}}_{11}} \quad \tau_{2}=\frac{m_{2} \widehat{\bar{C}}_{12}-\widehat{\bar{C}}_{22}+\left[\left(n_{1}^{2}+n_{1} n_{2}\right)-q^{2}\right] \bar{C}_{44}}{\widehat{\bar{C}}_{11}} \\
& \tau_{3}=\frac{\left(2 n_{1}+n_{2}\right) \bar{C}_{44}}{\widehat{\bar{C}}_{11}} q \quad \tau_{4}=\frac{n_{1} \widehat{\bar{C}}_{12}+\left(n_{1}+n_{2}\right) \bar{C}_{44}}{\widehat{\bar{C}}_{11}} \quad \tau_{5}=\frac{\widehat{\bar{C}}_{12}+\bar{C}_{44}}{\widehat{\bar{C}}_{11}} q \\
& \tau_{6}=\frac{\left(m_{2} \widehat{\bar{C}}_{12}-\widehat{\bar{C}}_{22}\right) n_{1}-\left(n_{1}+n_{2}\right) \bar{C}_{44}}{\widehat{\bar{C}}_{11}} \quad \tau_{7}=\frac{m_{2} \widehat{\bar{C}}_{12}-\widehat{\bar{C}}_{22}-\bar{C}_{44}}{\widehat{\bar{C}}_{11}} q \\
& \tau_{8}=\frac{\bar{e}_{21}}{\overline{\widehat{C}}_{11}} \quad \tau_{9}=\frac{\left(m_{4}+1\right) \bar{e}_{21}-\bar{e}_{22}}{\widehat{C}_{11}} \quad \tau_{10}=\left[\left(n_{1}^{2}+n_{1} n_{4}\right)-q^{2}\right] \frac{\bar{e}_{24}}{\widehat{\widehat{C}}_{11}} \\
& \tau_{11}=\left(2 n_{1}+n_{4}\right) \frac{\bar{e}_{24}}{\widehat{\bar{C}}_{11}} q \quad \tau_{12}=\frac{\alpha_{0}\left[\left(m_{1}+m_{2}+1\right) \bar{C}_{11}+2\left(m_{1}+m_{2}\right) \bar{C}_{12}-\bar{C}_{22}\right]}{\widehat{C}_{11}} \\
& \tau_{13}=\alpha_{0} \frac{\bar{C}_{11}+2 \bar{C}_{12}}{\widehat{\bar{C}}_{11}} \quad \tau_{14}=m_{2}+1 \quad \tau_{15}=\left(m_{2}+1\right)-\left[\left(n_{1}+n_{2}\right)-q^{2}\right] \frac{\widehat{\bar{C}}_{22}}{\bar{C}_{44}} \\
& \tau_{16}=\left(n_{2}+2\right) \frac{\widehat{\bar{C}}_{22}}{\bar{C}_{44}} q \quad \tau_{17}=n_{1}+\left(n_{1}+n_{2}\right) \frac{\widehat{\bar{C}}_{12}}{\overline{\bar{C}}_{44}} \quad \tau_{18}=\left(1+\frac{\widehat{\bar{C}}_{12}}{\bar{C}_{44}}\right) q \\
& \tau_{19}=\left(m_{2}+1\right) n_{1}+\left(n_{1}+n_{2}\right) \frac{\widehat{\bar{C}}_{22}}{\bar{C}_{44}} \quad \tau_{20}=\left(\left(m_{2}+1\right)+\frac{\widehat{\bar{C}}_{22}}{\bar{C}_{44}}\right) q \\
& \tau_{21}=\frac{\left(n_{1}+n_{4}\right) \bar{e}_{22}+n_{1} \bar{e}_{24}}{\bar{C}_{44}} \quad \tau_{22}=\frac{\bar{e}_{22}+\bar{e}_{24}}{\bar{C}_{44}} q \quad \tau_{23}=\left(m_{4}+2\right) n_{1} \frac{\bar{e}_{24}}{\bar{C}_{44}} \\
& \tau_{24}=\left(m_{4}+2\right) q \frac{\bar{e}_{24}}{\bar{C}_{44}} \quad \tau_{25}=\alpha_{0}\left(n_{1}+n_{2}\right) \frac{2 \bar{C}_{12}+\bar{C}_{22}}{\bar{C}_{44}} \\
& \tau_{26}=\alpha_{0} \frac{2 \bar{C}_{12}+\bar{C}_{22}}{\bar{C}_{44}} q \quad \tau_{27}=m_{5}+1 \quad \tau_{28}=\left[\left(n_{1}^{2}+n_{1} n_{5}\right)-q^{2}\right] \frac{\bar{\varepsilon}_{21}}{\bar{\varepsilon}_{22}} \\
& \tau_{29}=\left(2 n_{1}+n_{5}\right) q \frac{\bar{\varepsilon}_{21}}{\bar{\varepsilon}_{22}} \quad \zeta_{30}=-\frac{\bar{e}_{21}}{\bar{\varepsilon}_{22}} \quad \tau_{31}=\frac{\left(m_{4}+1\right) \bar{e}_{21}+\bar{e}_{22}}{\bar{\varepsilon}_{22}} \\
& \tau_{32}=\frac{m_{4} \bar{e}_{22}}{\bar{\varepsilon}_{22}} \quad \tau_{33}=\left(n_{1}+n_{4}\right) \frac{\bar{e}_{24}}{\bar{\varepsilon}_{22}} \quad \tau_{34}=\frac{\bar{e}_{24}}{\bar{\varepsilon}_{22}} q \\
& \tau_{35}=\frac{\left(m_{4}+1\right) n_{1} \bar{e}_{22}-\left(n_{1}+n_{4}\right) \bar{e}_{24}}{\bar{\varepsilon}_{22}} \quad \tau_{36}=\frac{\left(m_{4}+1\right) \bar{e}_{22}-\bar{e}_{24}}{\bar{\varepsilon}_{22}} q \\
& \tau_{37}=\frac{\left(m_{6}+1\right) \bar{g}_{21}+n_{6} \bar{g}_{22}}{\bar{\varepsilon}_{22}} \quad \tau_{38}=\frac{\bar{g}_{22}}{\bar{\varepsilon}_{22}} q \quad \tau_{39}=\frac{\bar{g}_{22}}{\bar{\varepsilon}_{22}}
\end{aligned}
$$

Also

$$
B=\frac{3\left(\nu_{u}-\nu\right)}{(1-2 \nu)\left(1+\nu_{u}\right)} \quad 0 \leqslant B \leqslant 1 \quad \phi=\frac{\gamma\left(B-k_{f}\right)}{B[(1-\alpha)+k]}
$$

where $k_{f}$ and $k$ are the bulk modulus of the fluid phase and the bulk modulus of the porouselastic medium under the drained condition, respectively

$$
M=\frac{2 G\left(\nu_{u}-\nu\right)}{\gamma^{2}(1-2 \nu)\left(1-2 \nu_{u}\right)}
$$

where $M$ and $\gamma$ are Biot's modulus, Biot's coefficient of the porouselastic medium, respectively. 
Using the boundary conditions (2.12) to determine the constants $A_{q_{1}}, A_{q_{2}}$

$$
\begin{aligned}
& \sum_{q=-\infty}^{\infty}\left[\left(S_{11} a^{\beta_{q_{1}}}+S_{12} \beta_{q_{1}} a^{\beta_{q_{1}}}-1\right) A_{q_{1}}+\left(S_{11} a^{\beta_{q_{2}}}+S_{12} \beta_{q_{2}} a^{\beta_{q_{2}}}-1\right) A_{q_{2}}\right] \mathrm{e}^{\mathrm{i} q \theta}=f_{1}(\theta) \\
& \sum_{q=-\infty}^{\infty}\left[\left(S_{21} b^{\beta_{q_{1}}}+S_{22} \beta_{q_{1}} b^{\beta_{q_{1}}}-1\right) A_{q_{1}}+\left(S_{21} b^{\beta_{q_{2}}}+S_{22} \beta_{q_{2}} b^{\beta_{q_{2}}}-1\right) A_{q_{2}}\right] \mathrm{e}^{\mathrm{i} q \theta}=f_{2}(\theta) \\
& \left(S_{11} a^{\beta_{q_{1}}}+S_{12} \beta_{q_{1}} a^{\beta_{q_{1}}}-1\right) A_{q_{1}}+\left(S_{11} a^{\beta_{q_{2}}}+S_{12} \beta_{q_{2}} a^{\beta_{q_{2}}}-1\right) A_{q_{2}}=\frac{1}{2 \pi} \int_{-\pi}^{\pi} f_{1}(\theta) \mathrm{e}^{-\mathrm{i} q \theta} d \theta \\
& \begin{array}{l}
\left(S_{21} b^{\beta_{q_{1}}}+S_{22} \beta_{q_{1}} b^{\beta_{q_{1}}}-1\right) A_{q_{1}}+\left(S_{21} b^{\beta_{q_{2}}}+S_{22} \beta_{q_{2}} b^{\beta_{q_{2}}}-1\right) A_{q_{2}}=\frac{1}{2 \pi} \int_{-\pi}^{\pi} f_{1}(\theta) \mathrm{e}^{-\mathrm{i} q \theta} d \theta \\
A_{q_{1}}=\frac{1}{2 \pi} \int_{-\pi}^{\pi} \frac{1}{\widehat{S}_{1}-\widehat{S}_{2}}\left[\left(S_{21} b^{\beta_{q_{2}}}+S_{22} \beta_{q_{1}} b^{\beta_{q_{2}}-1}\right) f_{1}(\theta)\right. \\
\left.\quad-\left(S_{11} a^{\beta_{q_{2}}}+S_{12} \beta_{q_{2}} a^{\beta_{q_{2}}-1}\right) f_{2}(\theta)\right] \mathrm{e}^{-\mathrm{i} q \theta} d \theta \\
A_{q_{2}}=\frac{1}{2 \pi} \int_{-\pi}^{\pi} \frac{1}{\widehat{S}_{1}-\widehat{S}_{2}}\left[\left(S_{11} a^{\beta_{q_{1}}}+S_{12} \beta_{q_{1}} a^{\beta_{q_{1}}-1}\right) f_{2}(\theta)\right. \\
\left.\quad-\left(S_{21} b^{\beta_{q_{1}}}+S_{22} \beta_{q_{1}} a^{\beta_{q_{1}}-1}\right) f_{1}(\theta)\right] \mathrm{e}^{-\mathrm{i} q \theta} d \theta \\
\widehat{S}_{1}=\left(S_{11} a^{\beta_{q_{1}}}+S_{12} \beta_{q_{1}} a^{\beta_{q_{1}}}-1\right)\left(S_{21} b^{\beta_{q_{2}}}+S_{22} \beta_{q_{2}} b^{\beta_{q_{2}}}-1\right) \\
\widehat{S}_{2}=\left(S_{11} a^{\beta_{q_{2}}}+S_{12} \beta_{q_{2}} a^{\beta_{q_{2}}}-1\right)\left(S_{21} b^{\beta_{q_{1}}}+S_{22} \beta_{q_{1}} b^{\beta_{q_{1}}}-1\right)
\end{array}
\end{aligned}
$$

\section{References}

1. Akbari Alashti R., Khorsand M., Tarahhomi M.H., 2013, Thermo-elastic analysis of a functionally graded spherical shell with piezoelectric layers by differential quadrature method, Scientia Iranica B, 20, 1, 109-119

2. Batifol C., Zielinski T.G., IchChou M.N., Galland M.-A., 2007, A finite-element study of a piezoelectric/poroelastic sound package concept, Smart Materials and Structures, 16, 168-177

3. Bowen C.R., Perry A., Lewis A.C.F., Kara H., 2004, Processing and properties of porous piezoelectric materials with high hydrostatic figures of merit, Journal of the European Ceramic Society, 24, 541-545

4. Ciarletta M., Scarpetta E., 1996, Some result on thermoelasticity for porous piezoelectric materials, Mechanics Research Communications, 23, 1, 1-10

5. Ding H.J., Wang H.M., Chen W.Q., 2004, Analytical solution of a special non-homogeneous pyroelectric hollow cylinder for piezothermoelastic axisymmetric plane strain dynamic problems, Applied Mathematics and Computation 151, 423-441

6. Hetnarski R.B., Eslami M.R., 2009, Thermal Stresses - Advanced Theory and Applications, Library of Congress Control No. 936149

7. Ivanov P.V., Eremkin V.V., Smotrakov V.G., Tsikhotskit E.S., 2002, Porous piezoelectric ceramics: materials for ultrasonic flaw detection and medical diagnostics, Inorganic Materials, $\mathbf{3 8}$, $4,408-410$

8. Jabbari M., Meshrini M., Eslami M.R., 2012, Nonaxisymmetric mechanical and thermal stresses in FGPPM hollow cylinder, Journal of Pressure Vessel Technology, 134, 061212-1 
9. Jabbari M., Meshinini M., Eslami M.R., 2016, Mechanical and thermal stresses in FGPPM hollow cylinder due to radially symmetric loads, Journal of Pressure Vessel Technology, 138, 011207-1

10. Li J.-F., TAkagi K., Ono M., PAn W., Watanabe R., 2003, Fabrication and evaluation of porous piezoelectric ceramics and porosity-graded piezoelectric actuators, Journal of the European Ceramic Society, 86, 1094-1098

11. Meshikini M., Firoozbakhsh K., Jabbari M., SelkGhafari A., 2017, Asymmetric mechanical and thermal stresses in 2D-FGPPMs hollow cylinder, Journal of Thermal Stresses, 40, 4, 448-469

12. Topolov V.Y., Turik A.V., 2001, Porous piezoelectric composites with extremely high reception, Technical Physics, 46, 9, 1093-1100

13. Zeng T., Dong X.L., Chen S.T., Yang H., 2007, Processing and piezoelectric properties of porous PZT ceramics, Ceramics International, 33, 395-399

14. ZiELINSKI T.G., 2010, Fundamentals of multi physics modeling of piezoporous-elastic structures, Archives of Mechanics, 62, 5, 343-378

Manuscript received January 4, 2017; accepted for print July 25, 2017 